\title{
Visibility and Proximity Based Risk Map of Earthwork Site Using Real-time Simulation
}

\author{
Faridaddin Vahdatikhaki ${ }^{a}$ and Amin Hammad ${ }^{\mathrm{b}}$ \\ ${ }^{a}$ Department of Building, Civil and Environmental Engineering, Concordia University, Canada \\ ${ }^{b}$ Concordia Institute for Information Systems Engineering, Concordia University, Canada \\ E-mail: vahdatifarid@yahoo.com, hammad@ciise.concordia.ca
}

\begin{abstract}
Approximately $75 \%$ of struck-by fatalities in the construction industry are reported to have been caused by heavy equipment. Researchers have addressed the need for the enhanced safety of earthwork equipment using different approaches. However, none of these approaches enables the equipment to accurately predict the operation of other pieces of equipment for a long-enough time window to find a collision-free path using path replanning. Accordingly, the present paper proposes a novel method to generate the risk map of an earthwork site considering the visibility and proximity based hazards. The proposed method integrates the pose and state data of different pieces of equipment with the prediction about the potential paths of equipment, which comes from near-realtime simulation and script-based path planning, to quantify the risk value associated with different portions of the site. The generated risk map can further be used by individual equipment to ensure that their future path is safe or to perform path replanning if required. The proposed method is implemented and tested in a case study. In the light of the results of a case study, it is found that the proposed method is providing a reliable basis for the safety analysis of earthwork sites
\end{abstract}

Keywords - Risk Map, Visibility and Proximity Hazards, Earthwork Equipment, Safety, Real-time Simulation

\section{Introduction}

The construction industry accounted for more than $17 \%$ of fatal work injuries, i.e., 806 counts of death, in the U.S. in 2012 [1]. A large range of construction projects involve earthwork, such as building foundations work, dam construction, airport construction, road construction, etc. [2]. However, the safety risks involved in earthwork operations are high due to the equipment-intensive nature of the work [3]. Occupational Safety and Health Administration (OSHA) reports that heavy equipment accounts for approximately $75 \%$ of struck-by fatalities in the construction industry, which is in turn the second cause of fatalities on construction sites after falling [4]. Hinze et al. [5] identify the use of adequate protective support systems as the primary method of preventing struck-by incidents on construction sites.

Researchers have addressed the need for the enhanced safety of earthwork equipment in two different streams. The first stream is to reduce the possibility of collisions between different pieces of equipment through applying more optimized planning and scheduling methods that consider the space requirements of various activities to avoid the dangerous proximities between different teams of equipment $[6,7,8,9,10]$. Such spaces are referred to in this paper as activity workspaces.

The second stream approached the problem from the monitoring point of view and exploits the real-time information regarding the pose, state and speed characteristics of the equipment to determine the spaces around the equipment that need to be safeguarded to ensure a safe operation within a short time window [11, $12,13,14,15,16,17]$, such spaces are called Dynamic Equipment Workspaces (DEW) in this paper.

While the activity workspaces can be used to perform the initial path planning of different equipment, such planning tends to lose its efficiency in the face of the multitude of unforeseen circumstances that may occur during a project. On the other hand, the dynamic equipment workspaces are merely designed as "the last line of defense" [16] to warn the operators against imminent collisions and, thus, are not able to provide the information and time window required for the path re-planning of the equipment. Accordingly, there is a need for a middle-level solution at the monitoring phase that is able to reliably predict the operation of the equipment for a long-enough time window to enable 
different pieces of equipment to adjust their planned paths and avoid collisions in near-real-time.

The emerging methods for near-real-time simulation of construction operations [18, 19, 20, 21] are providing the adequate inputs for a middle-level solution. Such methods are trying to build on the underlying logic of the operation, which is embedded in a simulation model, and use the data collected from the operation to continuously update its initial simulation model.

Stentz et al. [22] proposed a middle-level solution based on the prediction obtained from a parametric motion planning technique. Although the presented approach is efficient in finding a collision-free path for a single excavator, it is not able to consider a fleet of equipment and their interactions in determining the potential collisions between different pieces of equipment. Furthermore, in addition to distance-based risks, the blind spots of the equipment can place the safety of other equipment and crews at risk [23].

It is therefore imperative to develop a method to generate Look-Ahead Equipment Workspaces ( $L A E W s)$ that consider not only the proximity-based risks but also the visibility conditions of the site vis-à-vis the future states of the equipment. The spatial risk analysis leads to the generation of equipment risk maps that represent the risk distribution in the space around the equipment. These risk maps can then be used to generate the $L A E W s$ associated with a certain risk level.

Accordingly, the objectives of the present paper are: (1) Developing a novel method to generate equipment risk maps based on the integration of the proximitybased risks and visibility-based risks using the pose and state data of the equipment and the Near-Real-Time Simulation (NRTS); and (2) Generating $L A E W$ based on the equipment risk maps so that the resulting workspaces can be used to perform path re-planning when a potential collision is identified. This paper is a short version of a journal paper written by the authors [32].

\section{Proposed Method}

$L A E W s$ are generated for the purpose of look-ahead re-planning of equipment motions and are updated in near-real-time with an interval of $\Delta t$. The update interval is a function of the available computational power and the extent to which the future states of the equipment can be reliably predicted. Generally, the larger the value of $\Delta t$, the greater the chance of the potential changes in the predicted conditions, and thus the less the reliability of the generated $L A E W \mathrm{~s}$. In order to put the sensible value of $\Delta t$ into perspective, it is envisioned that it is most effective in a range between $10 \mathrm{~s}$ to $1 \mathrm{~min}$. While a value less than $10 \mathrm{~s}$ has the risk of being impractical for being too short for the planning of future motions, a value greater than $1 \mathrm{~min}$ reduces the reliability of the generated risk maps.

The proposed method is part of a multi-agent system $(M A S)$ that has been previously proposed by the authors to orchestrate the machine-level Location-based Guidance Systems (LGSs) technologies into a coherent project-level system committed to support earthwork operations towards the enhanced performance and safety of the overall project [24]. In the proposed $M A S$, several layers of agents are processing and managing the huge amount of collected sensory data into useful information that can be used in decision making at different operational levels. In a nutshell, Operator Agents (OAs) support the equipment operators and have the essential information about their current task, state and pose. In a construction site, often a group of equipment is teamed up to serve one particular operation, for instance several trucks and an excavator work together to move the earth. The team coordinators are supported by Team Coordinator Agents (TCAs), whose main objective is to track the progress of operations based on the data gathered from their subordinate OAs and to ensure safe and smooth delivery of the operations. Several layers of TCAs and a General Coordinator Agent (GCA) can be defined. Furthermore, these different types of agents will be fed by information agents who will provide the required data to the agents and frequently get updated based on the changes happening in the site as the construction progresses.

The flowchart of the proposed method for the generation of the $L A E W$ of one piece of equipment (equipment $q$ ) is shown in Figure 1. As shown in this figure, the input of this method comprises the sensory data, the equipment specifications and their accurate 3D models, the current pose and state data generated by the $O A$ of the equipment $q\left(O A_{q}\right)$, and future state data coming from the NRTS that is performed by the TCA. Additionally, the updated 3D model of the site, and the project detailed plan (including the location of different scheduled tasks, their time frame, and the site layout) are available through the Information Agent. Finally, given that a parametric motion planning is proposed to be used for the determination of the future motions of different pieces of equipment (discussed in Section 2.1.1), a set of heuristic rules that define the operation of a skilled operator is also required to be available to each $O A$.

The generation of $L A E W$ is based on the discretization of the entire site space into cells, and then calculating the risk associated with each cell given the future expected states of different pieces of equipment, which is performed by each $O A$. As shown in Figure 1, the pose data are used to identify the current state, which is then passed on to the TCA to perform the NRTS 
in order to generate the operational pattern of each $O A$. These data are then communicated with the $O A_{q}$ who will first integrate it with the updated $3 \mathrm{D}$ model of site and the equipment 3D model to situate the equipment in the virtual environment and then use the project plan,

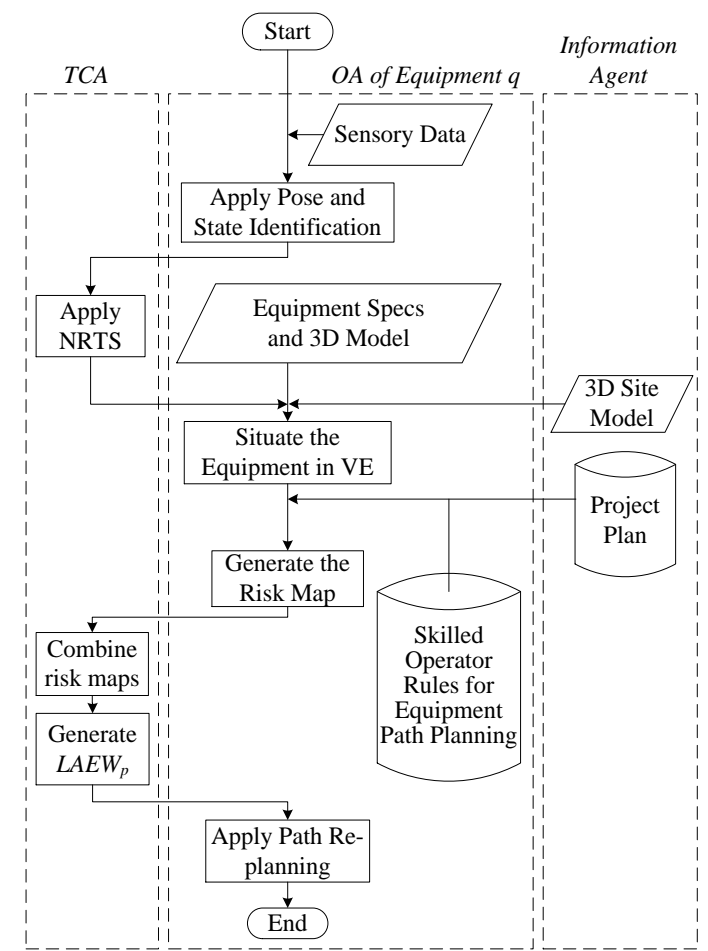

Figure 1. Flowchart of the Generation of $L A E W$

and the rules that govern the operation of the machine by a skilled operator to generate the risk map of the equipment, as will be explained in Section 2.1. Finally, the OAs transfer their individual risk maps to the TCAs who will first combine these risk maps and then use the tolerable risk level of each $O A$ to generate the $L A E W$. It should be highlighted that $L A E W_{p}$ for equipment $p$ is generated based on the combination of the risk maps from all pieces of equipment surrounding equipment $p$, excluding equipment $p$ itself. $L A E W_{p}$ can be used by the $O A_{p}$ to perform path re-planning, if required. Similarly, the path-replanning performed by the $O A_{q}$ at the end of flowchart shown in Figure 1 is realized through $L A E W_{q}$.

The previous works of the authors elaborated the method to obtain near-real time pose and state data [25, 21]. Therefore, the scope of the present paper begins from the point where all the input data are transferred to the virtual environment of the MAS for the determination of the motion path of the equipment. In the following sections, the $L A E W s$ of excavators are used to explain the proposed approach.

\subsection{Equipment Risk Map}

As shown in Figure 2, the first step for the generation of equipment risk maps is to identify the motion path of the equipment over the next $\Delta t$

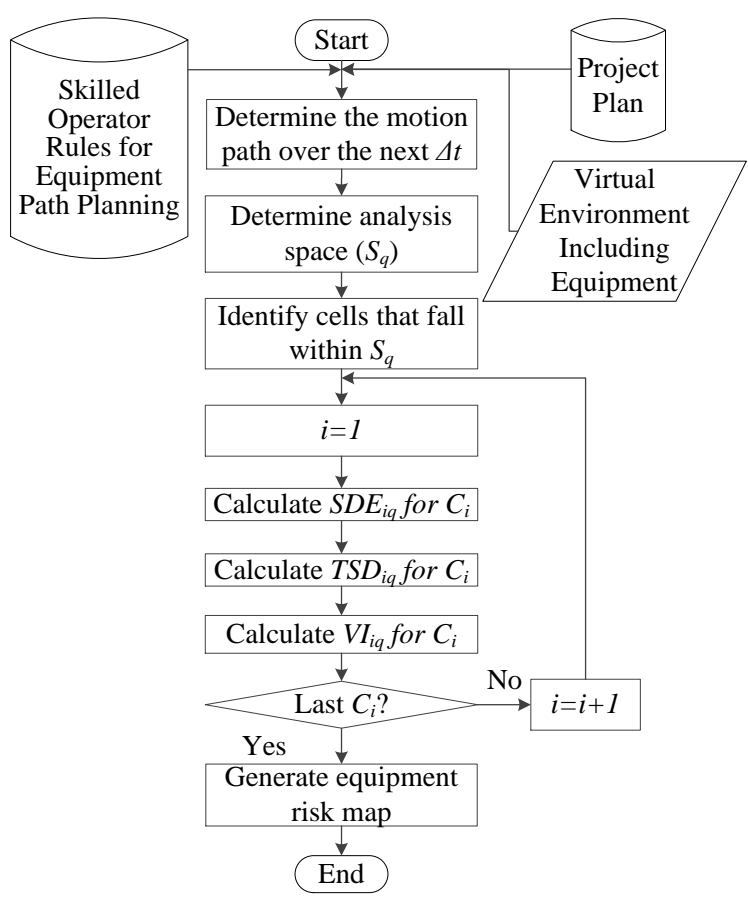

Figure 2. Flowchart of the Generation of Equipment Risk Map

(discussed in Section 2.1.1). Next, the $O A_{q}$ uses the generated path to identify the space that could be potentially impacted by the operation of the equipment in the next $\Delta t$. This space is referred to as the Analysis Space of equipment $q\left(S_{q}\right)$ and is discussed in Section 2.1.2. Furthermore, the cells that fall within $S_{q}$ are identified and reserved for the calculation of the risk pertinent to the operation of the equipment. Upon the completion of this step, three risk indices, namely Shortest Distance to Equipment $q\left(S D E_{i, q}\right)$, Time to Shortest Distance to Equipment $q\left(T S D_{i, q}\right)$ and Visibility Index of equipment $q\left(V I_{i, q}\right)$ are calculated for each cell $C_{i}$ in $S_{q}$. Subsequently, the $O A_{q}$ combines these risks factors to generate the overall risk map in $S_{q}$ (discussed in Section 2.1.3).

\subsubsection{Determining the Motion Path over $\Delta t$}

The first step in the generation of the equipment risk map is to determine the path of the equipment over the period of $\Delta t$. This step intends to combine the temporal data about the future states of equipment, coming from 
NRTS, with their corresponding spatial data, coming from a motion planning algorithm, to simulate the likely future paths of the equipment over $\Delta t$ in the virtual environment. This research adopts the parametric motion planning method [26, 27, 28] that combines inverse kinematic and rules from the motions executed by a skilled operator to find a smooth and realistic path for different pieces of equipment.

Figure 3 illustrates the flowchart for the integration of the NRTS data with the parametric motion planning. The inputs of this step are: (1) the dimensions of the equipment coming from the equipment specifications and 3D model, (2) the expected location of the equipment at different states (e.g., loading points and dumping points), (3) the sequence of upcoming states and their expected durations $\left(t_{k}\right)$ for different pieces of equipment based on the results of NRTS, (4) the current pose of the equipment, and (5) skilled operator rules that define a natural transition between different poses of the equipment.

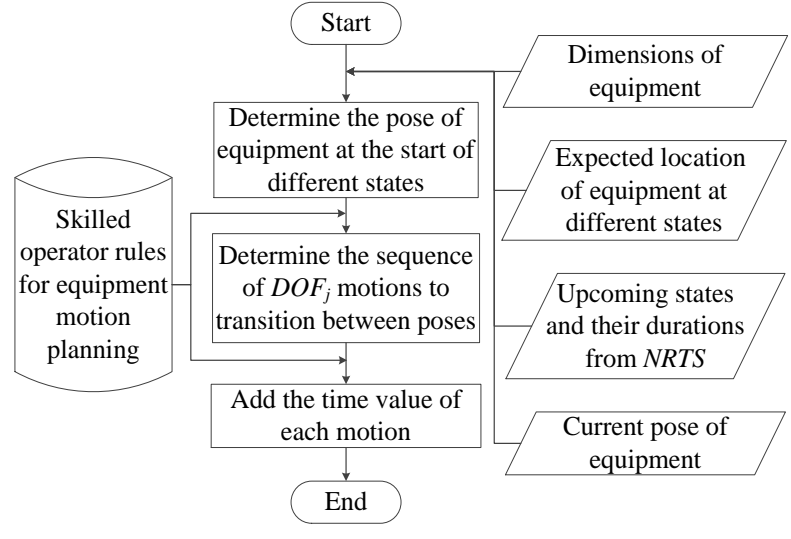

Figure 3. Flowchart of the Integration of NRTS data with Parametric Motion Planning

The first phase of this step is to identify the key poses that express the equipment at the start of different states. For this purpose, the results of NRTS are used to determine the future states of different equipment and

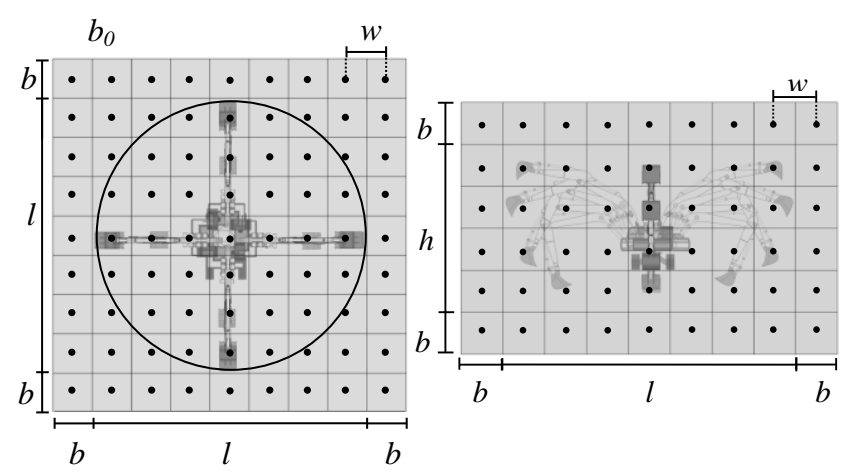

(a) (b) the time passed from the beginning of the present state, the time to be spent in the present state, and the expected durations of future states. Next, the expected location of the equipment at the determined states and the dimensions of equipment can be used to perform the inverse kinematic to identify these poses as proposed by Rowe [26], Kamat and Martinez [27], and Sarata et al. [28]. However, the sequence of these changes and the start time and the duration of changes in each $D O F$ are not known from the previous phase. Depending on the skill level of the operator, the changes in the values of several DOFs can happen with or without overlaps. This is because while a skilled operator is able to control multiple $D O F$ s at the same time, a novice operator most likely controls only one $D O F$ at a time. Through observing the skilled operator, the time at which a partial movement of a DOF starts and the duration of each partial movement can be determined.

\subsubsection{Determining the Analysis Space (S)}

The size of $S$ around the equipment is determined by the equipment dimensions, the equipment motion path, the equipment boundaries covering the entire space that can be occupied by the equipment through moving along any of its DOFs when performing stationary states (e.g., swinging or loading), and a buffer (b). The rationale behind the dependency of the analysis space on the motion path is that if the equipment is completely or partially in a traversal state (e.g., relocation) the analysis space needs to consider the space that is travelled by the equipment over $\Delta t$ in addition to the space determined by the equipment boundaries in stationary states. Figure 4 schematically represents this concept for an excavator. Figures 4(a) and (b) show the top and side views of an excavator, respectively, when it is moving along the extremes of its DOFs while it is stationary. When the equipment is partially or completely in traversal states, $S$ can elongate along the path of the equipment generated in the previous step, as shown in Figure 4(c). Finally, as shown in Figure 4(d), once the boundaries of $S$ are determined from the previous step, the cells from the global grid that are

Figure 4. (a) Top View of S for Excavator in Stationary State, (b) Side View of S for Excavator in Stationary State, (c) $\mathrm{S}$ for Excavator in Traversal State, and (d) Cells that Fall Within $S$ 
included in $S$ have to be determined.

\subsubsection{Determining the Risk Indices}

Once the analysis space is determined, the indices that determine the level of risk for each cell in $S$ can be calculated. $S D E_{i}, T S D_{i}$, and $V I_{i}$ are considered in this research. $S D E_{i, q}$ is defined as the distance between the center of the cell $C_{i}$ and the part of the equipment $q$ that moves closest to the cell during $\Delta t$. In other words, $S D E_{i, q}$ for $C_{i}$ is the smallest of the distances between motion paths of each part of equipment $q$ and the center of $C_{i}$. Figures 5(b), (c) and (d), schematically depict $S D E_{i, q}, T S D_{i, q}$, and $V I_{i, q}$ using the portion of the excavator operation shown in Figure 5(a). Figure 5(b) shows the different values of $S D E_{i, q}$ using color coding where red and green represent the extremes of the range, corresponding to low and high values of $S D E_{i, q}$, respectively. As shown in Figure 5(c), $T S D_{i, q}$ is defined as the temporal distance from $T_{\text {now }}$ to the time when the $S D E_{i, q}$ happens for cell $C_{i}$. The rationale is that the shorter the time to $S D E_{i, q}$, the greater the risk for the cell.

Finally, $V I_{i, q}$ is defined as an index representing the visibility of cell $C_{i}$ over the period of $\Delta t$ with respect to the blind spots of equipment $q$, considering static obstacles and the equipment $q$ itself. More precisely, $V I_{i, q}$ for cell $C_{i}$ can be defined as the total time the cell has not been in a blind spot of equipment $q$ over the period of $\Delta t$. A blind spot is defined as a portion of the equipment's surrounding space that remains invisible to the operator even if the operator has omni-directional view at his eyes' position. The assumption is that the skilled operator knows how to adjust his line of sight inside the cabin not to leave any visible space around the equipment overlooked. Also, since the calculation of risk indices are done by each $\mathrm{OA}$ in near real time, the locations of the other pieces of equipment are not considered in the determination of $V I_{i}$ of a cell. However, this is not an issue since the blind spot of equipment $q$ behind equipment $p$ is only hazardous if parts of equipment $q$ are planned to reach behind equipment $p$ over $\Delta t$, which is a very unlikely scenario.

\subsubsection{Generation of Equipment Risk Map}

Once all the indices are calculated, they need to be normalized so that the overall risk index associated with each cell can be measured in a range between zero and one; the value of zero representing a risk-free cell and the value of one representing a cell that is already colliding with the equipment and that remains invisible for the entire period of $\Delta t$, for example the cell that contains a part of the excavator superstructure during a swinging operation. Table 1 summarizes the three indices and their dimensions, initial ranges, and normalization method. In order to normalize $S D E_{i}$, it is divided by the largest value of $S D E_{i}$ within $\Delta t$ and then the result is subtracted from 1 , i.e., inverted so that the value of 1 represents the highest risk. Also to normalize $T S D_{i}$, and $V I_{i}$, their initially calculated values are divided by $\Delta t$ and the result is subtracted from 1 .

Equation (1) represents the method to combine all

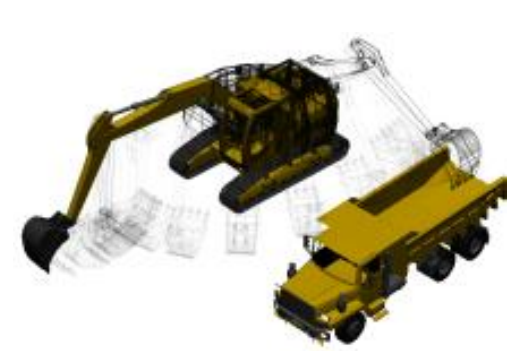

(a)

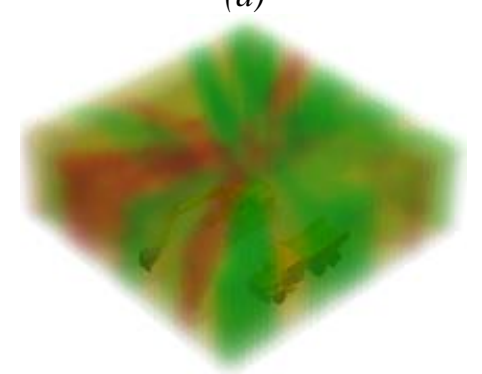

(d)

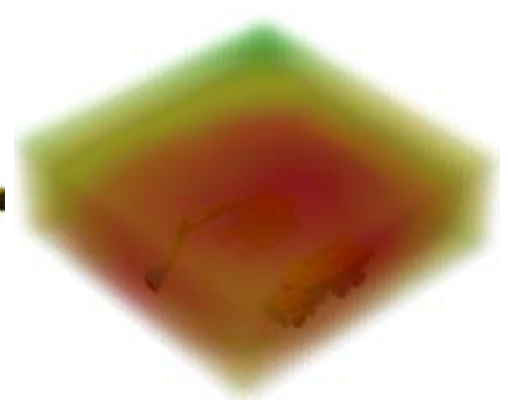

(b)

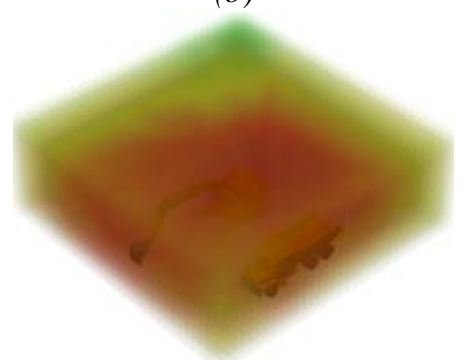

(e)

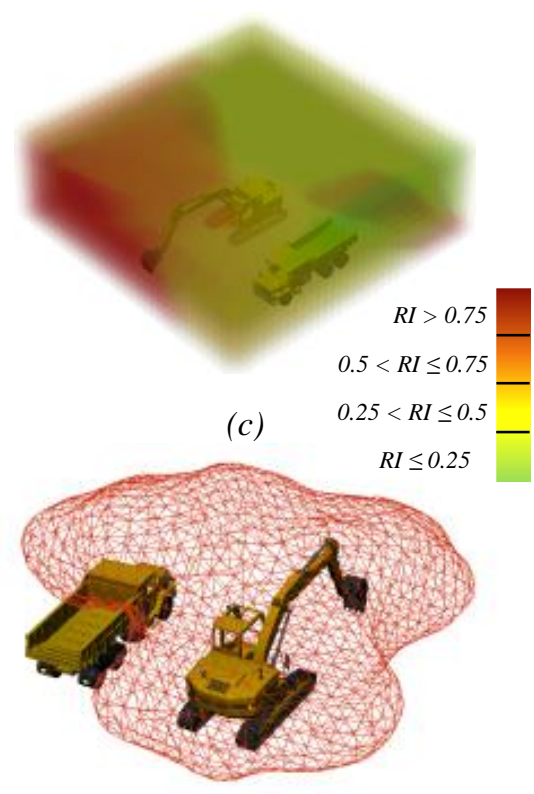

(f)

Figure 5. Schematic Representation of (a) Portion of Swinging in $S$, (b) $S D E_{i, q}$, (c) $T S D_{i, q},(d) V I_{i, q},(e)$ Combined Risk Map of the Excavator, (f) LAEW $W_{\text {truck }}$ 
the risk indices into one value considering different weights $\left(\mathrm{w}_{1}, \mathrm{w}_{2}, \mathrm{w}_{3}\right)$ for these indices. Although the weights are defined according to the risk attitude of the analyzer, it is recommended that $S D E_{i}$ and $V I_{i}$ are given the highest and lowest weights, respectively. This is due to the fact that while the consideration of $S D E_{i}$ is coming from the expected movements of the equipment, $V I_{i}$ is considered for the cases where the equipment may deviate from its expected movement and thus might pose a danger to crews and equipment in its blind spot.

Table 1. List of Indices and Their Normalization Method

\begin{tabular}{llll}
\hline Index & Unit & Range & Normalization \\
$S D E$ & Distance & {$\left[0 \sim S D E_{\text {max }}\right]$} & $1-\frac{S D E_{i}}{S D E_{\max }}$ \\
$T S D$ & Time & {$[0 \sim \Delta \mathrm{t}]$} & $1-\frac{T S D_{i}}{\Delta t}$ \\
$V I$ & Time & {$[0 \sim \Delta \mathrm{t}]$} & $1-\frac{V I_{i}}{\Delta t}$ \\
\hline
\end{tabular}

$R I_{i, q}=1-\frac{w_{1} \times \frac{S D E_{i, q}}{S D E_{q, \max }}+w_{2} \times \frac{T S D_{i, q}}{\Delta t}+w_{3} \times \frac{V I_{i, q}}{\Delta t}}{w_{1}+w_{2}+w_{3}}$

Where:

$R I_{i, q}$ : Overall risk index for cell $C_{i}$ of the risk map of equipment $q$

$w_{1}, w_{2}, w_{3}$ : Weights associated with $S D E_{i, q}, T S D_{i, q}$ and $V I_{i, q}$, respectively

$S D E_{q, \text { max }}$ : Maximum $S D E_{i, q}$ in $S_{q}$ over $\Delta t$

$\triangle t$ : Duration for the LAEW analysis

The outcome of this step is the risk map of the equipment over the period of $\Delta t$. Figure 5(e) shows an example of the excavator risk map based on the weight distribution of $(0.8,0.1,0.1)$.

\subsection{Generation and Application of LAEW $_{\mathrm{p}}$}

Once the risk maps of all pieces of equipment are generated by the OAs, the TCA uses them to generate the $L A E W_{p}$ for equipment $p$ for a specified risk level. As described in Section 2, it is important to re-emphasize that the risk maps are generated by the OAs of each piece of equipment (e.g. equipment $q$ ), and the TCA considers all the equipment that impact equipment $p$ to generate $L A E W_{p}$.

A TCA does not generate $L A E W_{p}$ solely based on the team of the equipment it is coordinating, given that there might be situations where a piece of equipment from one team is working in proximity to another team. Therefore, the TCA considers not only their subordinate OAs for the generation of $L A E W_{p}$, but also they communicate with other TCAs to identify OAs of other teams which are working in their proximity to incorporate their risk maps into $L A E W_{p}$. Nevertheless, the communication scheme between TCAs is out of the scope of the present paper and will be discussed in the future work of the authors.

The next step in the integration of the $L A E W_{p}$ is the readjustment of values of risk indices of multiple risk maps in view of the nature of interactions between various pieces of equipment. This is important because a piece of equipment does not pose the same level of risk to all other pieces of equipment. For instance, a truck that is being served by an excavator is more tolerant to the risks posed by that excavator compared to the risks posed by a loader from another team of equipment, given that the interaction and proximity between the truck and excavator is expected as part of the nature of operation they are performing. To consider this issue, the values of risk indices of the risk map of equipment $q$ that have to be considered to generate the $L A E W_{p}$ will be adjusted using $w_{q, p}$, which is a weight representing the level of significance of equipment $q$ for equipment $p$, as shown in Equation (2). The smaller the value of $w_{q, p}$, the more tolerant is equipment $p$ to the risks posed by equipment $q$.

$$
A R I_{i, q, p}=w_{q, p} \times R I_{i, q}
$$

Where:

$A R I_{i, q, p}$ : Adjusted overall risk index for cell $C_{i}$ of the risk map of equipment $q$ for the $L A E W$ of equipment $p$ $w_{q, p}$ : The weight associated with the level of significance of equipment $q$ for equipment $p$

$R I_{i, q}$ : Overall risk index for cell $C_{i}$ of the risk map of equipment $q$

After the determination of $A R I_{i, q, p}$ for all the equipment that affect equipment $p$, they are combined to generate $L A E W_{p} . L A E W_{p}$ is generated by considering the $A R I_{i, q, p}$ for the cells that belong only to one $S$, and the maximum of $A R I_{i, q, p}$ for the cells that belong to multiple $S$. Given that the initial path of the equipment is forecasted based on parametric motion planning in a deterministic manner, if a piece of equipment makes a decision based on the maximum overall risk indices of a space shared by multiple risk maps, it has already adapted itself to the worst case scenario.

Finally, $L A E W_{p}$ is generated as the envelope of all the cells with the $A R I_{i, q, p}$ equal to or greater than a specific risk level. Figure 5(f) shows the $L E A W_{\text {truck }}$ generated from the risk map of the excavator shown in Figure 5(e) with the risk level of 0.8. The $L E A W_{\text {truck }}$ can be used by the truck to analyze its initial future path in 
terms of whether or not it trespasses a high-risk spaces. The decision of which equipment needs to apply the path re-planning can be made based on the priorities of the operations of different pieces of equipment. However, once one piece of equipment performed path re-planning, steps explained in Sections 2.1.3 and 2.1.4 have to be repeated for that equipment and then the TCA should generate new $L A E W s$ for all the pieces of equipment that have lower priority than that equipment.

\section{Implementation and Case Study}

\subsection{Setting of the test}

A case study was conducted to verify and validate the proposed method for generating $L A E W s$. The data from a previously conducted lab test [21] were used to demonstrate the generation of risk maps and $L A E W s$ and their capacity to be used for equipment path re-planning

As shown in Figure 6, three pieces of remotelycontrolled scaled equipment were employed to simulate an earthwork operation where an excavator is dumping a hypothetical load to a dump truck, which in turn hauls the material to a dumping zone, dumps it, and then returns to the loading spot for the next load. The simulated operation consisted of four trips for the truck from the dumping zone to the loading zone, of which the first two loads were obtained from stockpile 1 and the next two from stockpile 2. A crane was placed near the excavator to simulate a congested site where several pieces of equipment are working in a tight area.

Ubisense's Ultra Wideband (UWB) technology [29] was used to track the equipment. The state and pose identification methods proposed in the authors' previous works [21, 25] have been deployed to provide the required input data. The pose correction method of UWB results in an update rate of is $1 \mathrm{~Hz}$ [25].

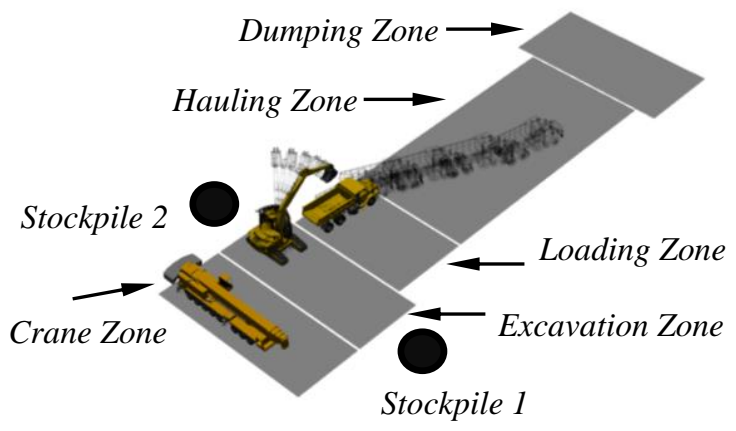

Figure 6. Schematic Layout of Simulated Site in the Case Study

\subsection{Implementation}

In order to demonstrate the applicability of $L A E W \mathrm{~s}$, a prototype system was developed, where Autodesk Softimage [30] was used as the virtual environment. It should be noted that the current state of the implementation focuses on generating the individual risk maps and combining them to generate the $L A E W \mathrm{~s}$. The 3D models of the equipment similar to those used in the case study were imported from Google's 3D warehouse [31]. The required modifications, including the placement of the operator's point of view in the cabin, which is used for the calculation of $V I$, and defining the tight-fitting bounding boxes, were done manually inside Softimage. The pose of the equipment was imported from the corrected UWB data using the pose estimation method [25], and the results of NRTS [21] were used to extract the temporal values of the upcoming states within $\Delta t$. The value of $\Delta t$ was set to $5.5 \mathrm{~s}$, which is a reasonable timeframe, given the scale of equipment and their corresponding speeds.

The visual programming embedded in Softimage is used to develop the tool for generating the global grid of the site and calculating the values of SDE, TSD, and VI. The movements of different parts of the truck and excavator in different states were modeled based on the operation of skilled operators as explained in Section 2.1.1. The initial paths generated for the truck and excavator based on the proposed method are shown in Figure 6. Once the different pieces of equipment are situated in the virtual environment and their respective movements over the next $\Delta t$ are simulated, the cells that fall within the analysis space of each equipment are determined based on its movement, as explained in Section 2.1.2. Then, while the operations of the equipment are simulated, the distances to the equipment and the visibility of each cell are calculated and recorded along with the time of the simulation. Upon the completion of the simulation, the aggregated values of the abovementioned parameters are used to calculate $S D E_{i}, T S D_{i}$, and $V I_{i}$ for the different pieces of the equipment, as explained in Section 2.1.3. These values are then normalized and combined through Equation (1), using the user-defined weights, to generate the risk map of each equipment. Finally, the risk levels assigned by the user are used to generate the $L A E W \mathrm{~s}$ of each piece of equipment.

\subsection{Results}

Figures 7(a) and (b) show the risk maps of excavator and truck, respectively, where only the cells with risk indices greater than zero are shown. The range of color from green to dark red shows the range of the risk index from zero to one. The distribution of weights for the 
parameters is set to $(0.8,0.1,0.1)$ for $S D E, T S D$, and $V I$, respectively. It can be discerned that the cells close to the motion path of the equipment have higher risk indices than those farther. Generally, the relative importance of spatial proximity against the temporal proximity should be used to determine the ratio of the weight of $S D E$ to the weight of TSD.

The inputs from Figures 7(a) and (b) are used to generate the $L A E W s$ corresponding to different risk levels. Figures 7(c) and (d) show $L A E W_{\text {truck }}$ and $L A E W_{\text {excavator, }}$ respectively, corresponding to the risk level of 0.8 for the same weights distribution. As stated in Section 2.1.4, the $L A E W_{\text {truck }}$ can be used by the truck to check potential collisions with its initial path, and to perform path re-planning if necessary.

\section{Conclusions and Future work}

This paper proposed a novel method for look-ahead equipment workspace for earthwork equipment that uses the predictive power of NRTS to evaluate the site safety based a number of parameters including $S D E, T S D$, and $V I$. This method enables different pieces of equipment to ensure that their initially planned paths are collisionfree, or alternatively adjust their path planning to avoid potential collisions. The discretization of space is applied to decompose the earthwork site into a number of cells in a virtual environment. Next, NRTS and parametric motion planning are used to predict the future motions of different pieces of equipment over the period of $\Delta t$. Using this information, the risk index of each cell is evaluated in terms of SDE, TSD, and VI. The generated risk map is then leveraged to determine the $L A E W \mathrm{~s}$ of the equipment corresponding to a given risk level. A case study was conducted to validate the proposed method.

In light of the results of the case study, it can be concluded that: (1) the proposed method is providing a reliable basis for the generation of the risk maps of earthwork equipment, using the expected pose and state and considering the proximity-based and visibilitybased risks; and (2) the risk maps can be combined to generate $L A E W \mathrm{~s}$ with different risk levels that can be used by different equipment and crews based on the varying levels of risk they can tolerate to adjust their initial paths.

The authors will be focusing on the following aspects of the proposed method in the future: (1) applying advanced path planning algorithms to enable the generation of the equipment motion paths in cases where the parametric motion planning method may not work because of the presence of many obstacles; and (2) using a probabilistic risk assessment method to consider the uncertainties of the predicted motion paths of the equipment.

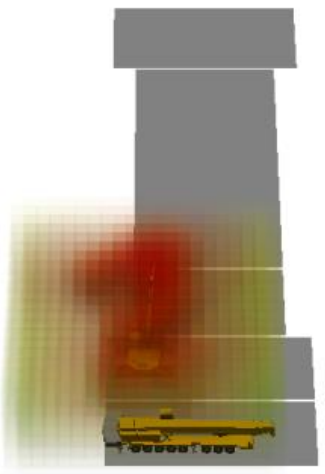

(a)

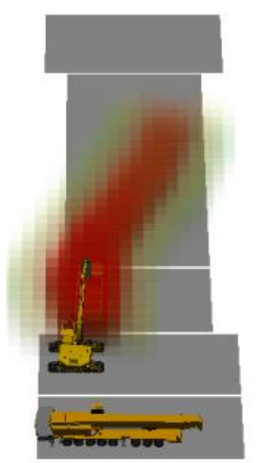

(b)

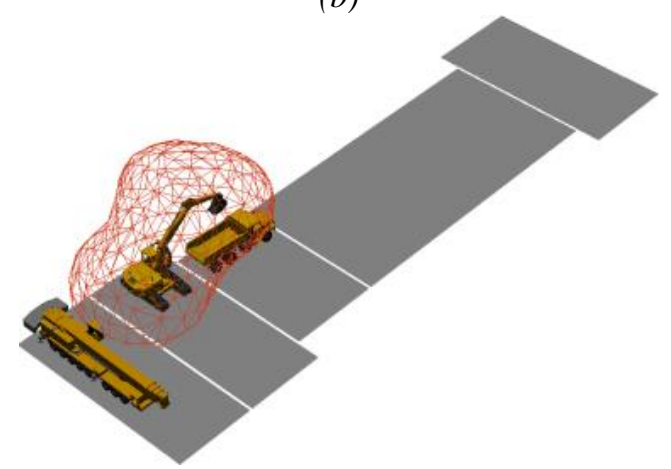

(c)

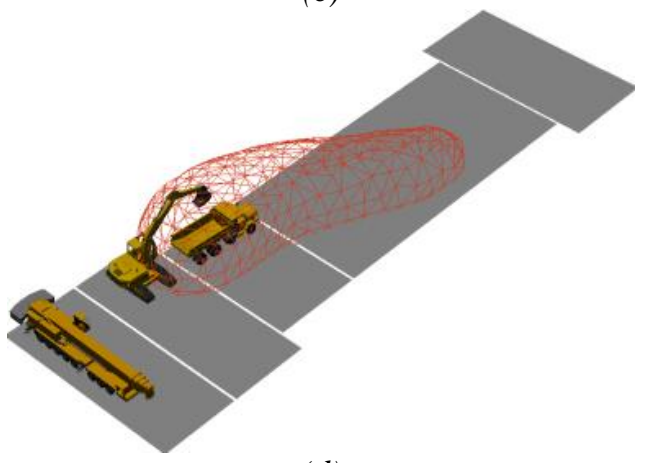

(d)

Figure 7. Risk Maps for (a) Excavator and (b)

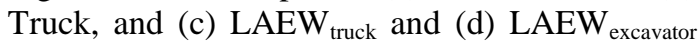
with Risk Level of 0.8 


\section{References}

[1] CFOI, Injuries, Illnesses, and Fatalities. Online http://www.bls.gov/iif/oshwc/cfoi/cfch0011.pdf, 2014

[2] Shi J. and AbouRizk S. S. An automated modeling system for simulating earthmoving operations. Computer-Aided Civil and Infrastructure Engineering, 13(2):121-130, 1998.

[3] Peurifoy R. L., Schexnayder C. J., Shapira A. and Schmitt R. L. Construction planning, equipment, and methods, New York: McGraw-Hill, 2011.

[4] OSHA, OSHA Construction eTool. Online: https://www.osha.gov/SLTC/etools/construction/st ruckby/mainpage.html, 2014

[5] Hinze J., Huang X. and Terry L. The nature of struck-by accidents. Journal of Construction Engineering and Management, 131(2):262-268, 2005.

[6] Mallasi Z. and Dawood N. Assessing space criticality in sequencing and identifying execution patterns for construction activities using VR visualisations. In ARCOM doctoral research workshop: Simulation and modelling in construction, 2001.

[7] Chavada R., Dawood N. N. and Kassem M. Construction workspace management: the development and application of a novel $\mathrm{nD}$ planning approach and tool. ITcon, 17:213-236, 2012.

[8] Tantisevi K. and Akinci B. Automated generation of workspace requirements of mobile crane operations to support conflict detection. Automation in Construction, 16(3):262-276, 2007.

[9] Hammad A., Zhang C., Al-Hussein M. and Cardinal G. Equipment workspace analysis in infrastructure projects. Canadian Journal of Civil Engineering, 34(10):1247-1256, 2007.

[10] Moon H., Kim H., Kim C. and Kang L. Development of a schedule-workspace interference management system simultaneously considering the overlap level of parallel schedules and workspaces. Automation in Construction, 39: 93-105, 2013.

[11] Burns R. L. Dynamic Safety Envelope For Autonomous-Vehicle Collision Avoidance System. U.S. Patent, US 6393362, 2002.

[12] Carbonari A., Giretti A. and Naticchia B. A proactive system for real-time safety management in construction sites. Automation in Construction,
20(6):686-698, 2011.

[13] Zhang C. and Hammad A. Improving lifting motion planning and re-planning of cranes with consideration for safety and efficiency. Journal of Advance Engineering Informatics, 26(2), 2012.

[14] Guenther N. and Salow H. Collision avoidance and operator guidance innovating mine vehicle safety. In Proceedings of the Queensland Mining Industry Health \& Safety Conference, Townsville, 2012.

[15] Wu H., Tao J., Li X., Chi X., Li H., Hua X., Yang R., Wang S. and Chen N. A location based service approach for collision warning systems in concrete dam construction. Safety Science, 51(1):338-346, 2013

[16] Zolynski G., Schmidt D. and Berns K. Safety for an Autonomous Bucket Excavator During Typical Landscaping Tasks. New Trends in Medical and Service Robots, 20: 357-368, 2014.

[17] Vahdatikhaki F. and Hammad A. Dynamic Equipment Workspace generation for improving earthwork safety using real-time location system. Advanced Engineering Informatics, (Under Review), 2015.

[18] Lu M., Dai F. and Chen W. Real-time decision support for planning concrete plant operations enabled by integrating vehicle tracking technology, simulation, and optimization algorithms. Canadian Journal of Civil Engineering, 912-922, 2007.

[19] Akhavian R. and Behzadan A. H. An integrated data collection and analysis framework for remote monitoring and planning of construction operations. Advanced Engineering Informatics, 26(4):749-761, 2012.

[20] Song L. and Eldin N. N. Adaptive real-time tracking and simulation of heavy construction operations for look-ahead scheduling. Automation in Construction, 27:32-39, 2012.

[21] Vahdatikhaki F. and Hammad A. Framework for near real-time simulation of earthmoving projects using location tracking technologies. Automation in Construction, 42:50-67, 2014.

[22] Stentz A., Bares J., Singh S. and Rowe P. A robotic excavator for autonomous truck loading, Autonomous Robots, 7(2):175-186, 1999.

[23] Soltani A. R., Tawfik H., Goulermas J. Y. and Fernando T. Path planning in construction sites: performance evaluation of the Dijkstra, A*, and GA search algorithms. Advanced Engineering 
Informatics, 16(4):291-303, 2002.

[24] Hammad A., Vahdatikhaki F. and Zhang C. A novel integrated approach to project-level automated machine control/guidance systems in construction projects. ITcon, 162-181, 2013.

[25] Vahdatikhaki F., Hammad A. and Siddiqui H. Optimization-based Excavator Pose Estimation Using Real-time Location Systems. Automation in Construction, (accepted with modifications), 2015.

[26] Rowe P. S. Adaptive motion planning for autonomous mass excavation. PhD thesis, Carnegie Mellon University, 1999.

[27] Kamat V. R. and Martinez J. C. Dynamic 3D visualization of articulated construction equipment. Journal of computing in civil engineering, 19(4):356-368, 2005.

[28] Sarata S., Koyachi N., Tsubouchi T., Osumi H., Kurisu M. and Sugawara K. Development of autonomous system for loading operation by wheel loader. In Proceedings of the 23rd International Symposium on Automation and Robotics in Construction, ISARC, 2006.

[29] Ubisense, Online: http://www.ubisense.net/en/, 2013.

[30] AutoDesk, “AutoDesk SoftImage," Online: http://www.autodesk.com/products/softimage/over view, 2014.

[31] Google 3D Warehouse. Online: https://3dwarehouse.sketchup.com/?redirect, 2014.

[32] Vahdatikhaki F., Hammad A. Risk-based Lookahead Workspace Generation for Earthwork Equipment Using Near Real-time Simulation. Automation in Construction, (accepted with modifications), 2015. 